\title{
El uso de los biomodelos didácticos en las ciencias veterinarias: Una revision.
}

\section{The Use of Teaching Biomodels in Veterinary Sciences: Review}

\section{O uso dos biomodelos didáticos nas Ciencias Veterinarias: Uma revisão.}

\author{
David Fernando Balaguera Quinche ${ }^{1 凶}$, Javier Arturo Vesga Castillejo ${ }^{1}$, Andrés Laureano Burgos Guzmán ${ }^{1}$, \\ Jeyson Alejandro Simbaqueva Peña ${ }^{1}$, Jhoan Sebastián Rodríguez Rodríguez ${ }^{2}$, Juan David Córdoba Parra ${ }^{1}$
}

1 Facultad de Medicina Veterinaria, Fundación Universitaria Agraria de Colombia UNIAGRARIA, Bogotá, Colombia. balaguera.david@uniagraria.edu.co, vesga.javier@uniagraria.edu.co, burgos.andres@uniagraria.edu.co, simbaqueva.jeyson@ uniagraria.edu.co, cordoba.juan@uniagraria.edu.co

2 Facultad de Ingeniería, Fundación Universitaria Agraria de Colombia UNIAGRARIA, Bogotá, Colombia, rodriguez.jhoan@uniagraria.edu.co

Recibido: 9 de noviembre de 2020 Corregido: 13 de abril de 2021 Aceptado: 21 de mayo de 2021

\begin{abstract}
Resumen
Los modelos didácticos se han empleado a través del tiempo como un medio para el entendimiento de la medicina humana y animal; especialmente en asignaturas como: anatomía, fisiología, cirugía y patología, las cuales son de mayor relevancia en la formación del profesional en medicina. Estos modelos son maquetas artificiales, tridimensionales. Buscan una aproximación a la morfología y función de un organismo; ayudan a su exploración y, en lo posible, un reemplazo para disminuir las prácticas con experimentación animal. Este artículo se elaboró mediante la búsqueda bibliográfica en diversas fuentes. También, se presenta ejercicios didácticos propios, de los autores, para resaltar algunos aspectos importantes del uso de los biomodelos desde diferentes disciplinas de la medicina veterinaria. Asimismo, diferentes puntos de vista para su implementación como herramienta que promueva el proceso de enseñanza - aprendizaje en los estudiantes.
\end{abstract}

Palabras clave: Biomodelo, aprendizaje, enseñanza, didáctica, veterinaria.

\begin{abstract}
Didactic models have been used throughout many years as a means for understanding human and animal medicine, especially in subjects such as anatomy, physiology, surgery, and pathology that are central to the training of the medical professional. These models are three-dimensional artificial models that seek an approximation to the morphology and function of an organism, help its exploration and, if possible, a replacement to reduce practices with animal experimentation. This literature review was constructed by searching different sources, and the authors' own didactic exercises will also be presented to highlight some important aspects of the use of didactic biomodels from different disciplines of veterinary medicine and also different points of view for their implementation as a tool to promote the teaching-learning process in students.
\end{abstract}

Keywords: biomodel, learning, teaching, didactics, veterinary

$凶$ Autor de correspondencia: balaguera.david@uniagraria.edu.co 


\begin{abstract}
Resumo
Os modelos didáticos têm sido usados ao longo do tempo como um meio para a compreensão da medicina humana e animal, especialmente em disciplinas como anatomia, fisiologia, cirurgia e patologia que são centrais para a formação de profissionais em medicina. Esses modelos são maquetas artificiais tridimensionais que buscam uma aproximação à morfologia e função de um organismo, auxiliam na sua exploração e, se possível, um substituto para reduzir as práticas de experimentação animal. Este artigo foi construído através de um levantamento bibliográfico em diferentes fontes e também são apresentados exercícios didáticos dos próprios autores para destacar alguns aspectos importantes do uso de biomodelos a partir de diferentes disciplinas da medicina veterinária, bem como, diferentes pontos de vista para sua implementação como ferramenta para promover o processo de ensino-aprendizagem dos estudantes.
\end{abstract}

Palavras-chave: Biomodelo, aprendizagem, ensino, didática, veterinária.

\title{
Introducción
}

Los modelos animales se han empleado, desde la antigüedad, para el entendimiento de procesos fisiológicos, anatómicos, patológicos y como línea de base para estudios en humanos (Gómez et al., 2010). El uso de los animales de experimentación es uno de los pilares fundamentales en el desarrollo de las ciencias de la salud. Ha permitido un avance cada vez más acelerado sobre los conocimientos de tipo biológico (Villamizar \& Aquino, 2016). En las investigaciones biomédicas, se precisa la utilización de los animales de laboratorio como biomodelos naturales o inducidos, con diversas enfermedades, los cuales ayudan al estudio y la comprensión de la patogenia y posibilidades de tratamiento. (Lujan et al., 2000) Sin embargo, se ha incrementado la preocupación por su bienestar, como se conoce, teniendo en cuenta los principios de las 3R (reemplazo, reducción y refinamiento).

Al realizar un recuento histórico de la exploración del organismo y sus diferentes partes, se encuentra a Acmaeon de Crotona, el cual demostró la función del nervio óptico, al seccionarlo provoca la ceguera del animal. El Corpus Hipocraticus, de la escuela de Hipócrates, describe una experiencia en la que, para comprobar el proceso de la deglución, se seccionó la garganta a un cerdo. Herófilo (330-250 a.C.) demostró la diferencia funcional entre nervios y tendones con estudios anatómicos de animales. El Grande ordenó, a su médico, efectuar ensayos en animales, de la acción tóxica de los venenos y su protección mediante antídotos. Galeno practicó técnicas de disección en abundancia, realizándolas en distintas especies. En sus estudios no solo se describe la anatomía, sino que se interpreta gran cantidad de funciones, como la de los pulmones y la del corazón (Izaguirre et al., 2001), entre otros. Todos estos hechos históricos nos muestran que la experimentación animal fue la primera práctica realizada por el hombre para develar los secretos internos del cuerpo, mucho antes de intentar representar y replicar sus componentes biológicos.

Desde aquel momento, cuando inicia la documentación de las primeras aproximaciones, para representar estructuras, como las esculturas anatómicas, conocidas inicialmente como Ceras Anatómicas (Forero, 2016), inicia la finalidad de transmitir estas características a los aprendices para mejorar el conocimiento. Desde siempre, el uso de animales en docencia ha tenido un alto valor, debido a la posibilidad de poder observar y conocer los órganos y tejidos, así como reconocer las reacciones corporales en pacientes reales en algunas materias como: farmacología, fisiología o anatomía. Se realiza prácticas de laboratorio que implican el uso de animales o tejidos. En muchos casos, los objetivos, de estas clases, están poco definidos; en otros, se habla de la necesidad de aprender 
habilidades prácticas y de laboratorio, relacionadas con la manipulación de animales, la disección o cirugía. También, se indica que este tipo de prácticas son importantes para reforzar los conocimientos adquiridos en clases teóricas; aprender a hacer mediciones; toma de datos, su análisis; representación e interpretación. También, para adquirir habilidades relacionadas con la comunicación oral y escrita.

El intento por mejorar la formación, en el ámbito de la medicina, es un tema que incide en todas las asignaturas impartidas en la academia, como lo afirma (Gonzales et al., 2015) “continuamos con la búsqueda de acciones que nos lleven a un nuevo rumbo de trabajo con panoramas frescos y alentadores" para nuestros estudiantes. La educación médica no es mundo que se aleje de este intento, dado que puede ser un espacio para la combinación de métodos de enseñanza y elementos interactivos (Friederichs et al., 2013). Usualmente, las lecciones han sido criticadas por ser monótonas; en las cuales los estudiantes son aprendices pasivos, sumado a que los estudios, con libros de texto, parecen proporcionar solo una comprensión limitada en los estudiantes (Kuebler et al., 2007). Sin embargo, acá se implementen diferentes didácticas. No es posible eliminar, por completo, las conferencias. Estas sirven como medio para presentar, a los estudiantes, conceptos complejos. Por consiguiente, buscar herramientas complementarias a las clases magistrales puede ser un medio para mejorar el aprendizaje y la enseñanza de asignaturas como: fisiología, anatomía, cirugía y patología.

A través del tiempo, se ha utilizado la metodología tradicional de enseñanza, la cual consiste en clases magistrales teóricas y sesiones de laboratorio, con el fin de "poner en práctica la teoría”. Actualmente, está demostrado que esta metodología favorece, en los estudiantes, el conocimiento primario (capacidad para memorizar conceptos) y la comprensión secundaria (capacidad de entender los conceptos); pero ¿se podrá utilizar otras metodologías para conseguir los mismos resultados, o tal vez mejorar otras habilidades de pensamiento superior? En los últimos años, se ha implementado la didáctica de construcción de modelos a escala (por ejemplo, anatómicos) con el fin de mejorar el aprendizaje de las ciencias. ¿Puede ser útil esta implementación en estudiantes a nivel de pregrado en medicina veterinaria? En este capítulo se expone algunos aspectos importantes del uso de los modelos didácticos desde las asignaturas de: fisiología, anatomía, cirugía y patología; asimismo, su implementación como herramienta para promover el proceso de enseñanza - aprendizaje en los estudiantes de Medicina Veterinaria.

\section{Los modelos didácticos en fisiología}

Los modelos didácticos se han utilizado durante mucho tiempo como herramientas educativas, dado que pueden ser más útiles que las imágenes bidimensionales en el aprendizaje y la retención del contenido de anatomía y fisiología. Según (Lujan et al. 2013), es una excelente manera de proporcionar una actividad basada en la investigación, colaboración y resolución de problemas que mejora el aprendizaje, promueve la curiosidad, la objetividad y el uso del razonamiento científico. Según Lenis \& Arango (2011), los modelos hechos por los mismos estudiantes promueven las capacidades analíticas, argumentativas o de innovación; adicionalmente favorecen el desarrollo de la imaginación. Además, fomenta la creatividad, la innovación y el emprendimiento en los estudiantes. Rodenbaugh et al. (2012) afirman que la construcción de modelos físicos brinda oportunidades para que el estudiante piense acerca de la información, se involucre en el proceso, desarrolle una comprensión funcional del material y use las habilidades de razonamiento (Dicarlo, 2013). Estas cualidades podrían ser de utilidad en la enseñanza - aprendizaje de la fisiología; cambiar un poco el Modelo tradicional, el cual se centra en la transmisión de conocimiento en un solo sentido, desde el profesor 
hacia el estudiante. La implementación de los modelos didácticos en fisiología puede ser una herramienta más para este fin.

Al realizar la búsqueda, sobre la implementación de los modelos didácticos en fisiología, se encuentra resultados positivos. Algunos se enuncian a continuación:

- Al implementar un modelo didáctico sobre la fisiología renal, la mayoría de los estudiantes estuvo de acuerdo en que el modelo era fácil de entender, mejoró su conocimiento y apreciación de la importancia de filtración y recomendaría el modelo a sus compañeros; además, la mayoría de ellos, [63 de 76 estudiantes (83\%)] preferiría el uso del modelo en lugar del estudio de los materiales escritos (Gookin et al., 2010).

- Al utilizar un modelo didáctico, para ejemplificar los movimientos oculares, todos los estudiantes del grupo sintieron que el modelo era muy útil para comprender y entender los ejes de los movimientos. Algunos comentarios específicos fueron: "Muy bien”, "Lo entendí muy bien”, "Es muy simple”, "Nunca pensé que fuera tan fácil” y "Por favor, usen estos modelos para otros temas también”. De acuerdo con los investigadores, la atención del estudiante podía ser captada en un minuto (Satheesha \& Soumya, 2009).

- Como un intento de explicar la fisiología de la contracción muscular, se creó un modelo de fibras a partir de materiales simples, como cuerdas y pelotas. La naturaleza tridimensional de los modelos ayudó a los estudiantes a comprender y apreciar los aspectos estructurales del músculo esquelético. Hubo un 51.8\% ( $n=83)$ que consideraron el uso de modelos "muy útil”; 42.2\% los encontró “útiles”; mientras que el 4.8\% fue neutral en su respuesta. El 1.2\% de los estudiantes encontró que los modelos eran "confusos". No hubo quienes consideraran el uso de modelos "muy confuso". De acuerdo con el investigador, los estudiantes disfrutaron estas intervenciones, haciendo que el proceso de aprendizaje sea tanto informativo como agradable (Anandit et al., 2018).

- Cuando se implementó un modelo para explicar los procesos del ciclo cardíaco, los estudiantes comentaron que este les ayudó a recordar los conceptos; los mantuvo despiertos y la sesión fue interesante. De acuerdo con el investigador, esta actividad simple se puede utilizar para hacer una conferencia más animada (Sajal et al., 2018).

- Al utilizar un modelo sobre el sistema respiratorio, aumentó la comprensión de la mecánica respiratoria en un $70 \%$, en comparación con la extensa enseñanza didáctica previa de los estudiantes; los cuales, en sus comentarios individuales, externaron que "este es un buen modelo para comprender muchos estados patofisiológicos"; que "sin este modelo nunca hubieran entendido las diferentes características del sistema respiratorio". De acuerdo con el investigador, este modelo fomenta la comprensión de las interacciones y puede mejorar la comprensión de la fisiología respiratoria (Kuebler et al., 2007).

- En Colombia, particularmente en las universidades, algunos modelos son desarrollados por el propio interés de profesores e investigadores, o por los propios estudiantes interesados en alguna temática en particular, con materiales poco duraderos en el tiempo como: yeso, plastilinas, espumas de poliuretano, entre otras.

- En la Fundación Universitaria Agraria de Colombia, por iniciativa del profesor David Fernando Balaguera (autor principal), en la asignatura de fisiología, se exponen los proyectos de aula, basados en la creación de modelos didácticos. A continuación, unos ejemplos (Fig 1). 

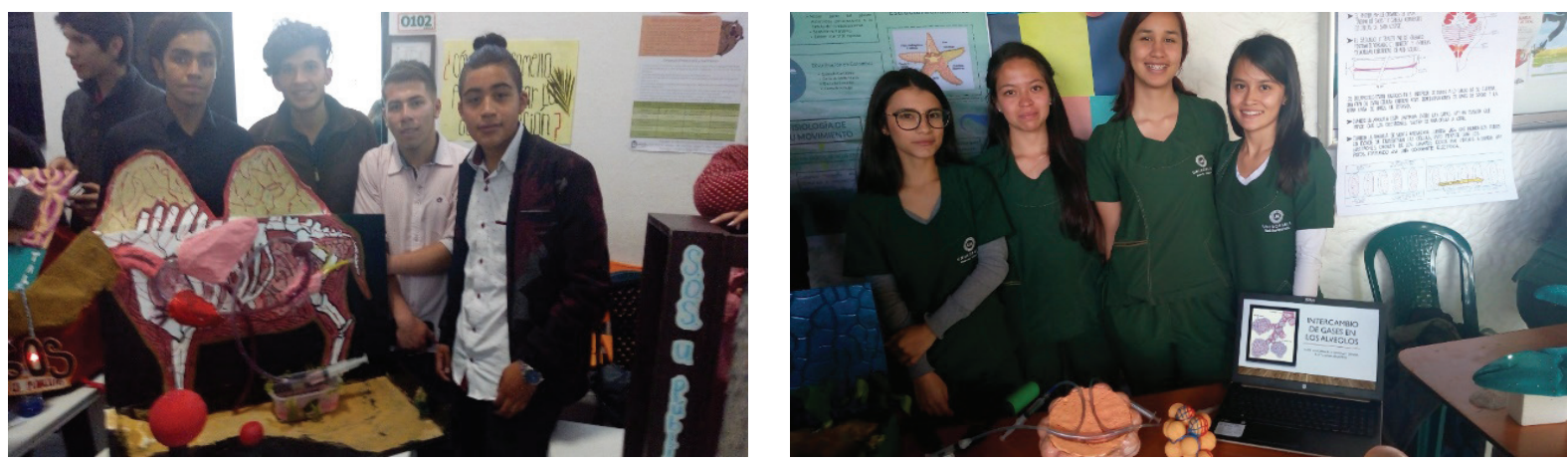

Figura 1. Estudiantes representando biomodelo. (Fuente autor)

\section{Los modelos didácticos en anatomía}

Actualmente, la principal herramienta de estudio macroscópica es por anatomía comparativa, en la cual se investiga la forma y el desarrollo del cuerpo animal a través de la analogía entre diferentes especies (Massari et al., 2019). Un conocimiento sólido sobre la anatomía es fundamental para las diversas áreas de la salud. Este hecho está directamente relacionado con la importancia de los métodos utilizados en el proceso de enseñanza-aprendizaje de anatomía para la formación de los estudiantes.

Un factor importante es que, entre todos los recursos didácticos, los que han demostrado ser más eficientes hasta ahora, en el aprendizaje de la anatomía, son los cadáveres. Esto se debe al contacto con estructuras reales, proporcionando una asimilación precisa de sus particularidades (De Bona, 2019; Lee, 2010). Por otra parte, para el estudio de la anatomía, se ha implementado varias herramientas y técnicas que buscan, principalmente, imitar las estructuras biológicas estáticas, especialmente para uso educativo. Por ejemplo, la digitalización 3D, la cual consiste en la generación de un modelo informático tridimensional de un objeto. El modelo digital puede procesarse en un sistema informático no solamente para generar imágenes, sino también para realizar cálculos, estudiar sus propiedades conformacionales y estructurales, editarlo o imprimirlo (Nobile, 2016). La creación de nuevas técnicas rápidas para la elaboración de prototipos, el bajo costo de las impresoras 3D, así como la aparición de nuevos softwares, para el análisis de las imágenes, han permitido la creación de modelos de huesos en 3D, cuya aplicación es posible en el campo de la enseñanza de la anatomía en muchas universidades de ciencias animales (Massari et al., 2019). Los modelos resultantes de la creación rápida de prototipos, que reproducen morfológicamente estructuras del cuerpo, han recibido el nombre de biomodelos. Un ejemplo claro ocurre en osteología. Hoy, los biomodelos de esqueletos caninos y equinos reportan el uso de la impresión 3D para producirlos en masa (Lee 2010; Massari et al., 2019). Estas técnicas han revolucionado el escenario de los procedimientos quirúrgicos. Tienen un modelo de la estructura anatómica del paciente, en la cual se realizará la cirugía; proporciona al cirujano una planificación más detallada. Otra ventaja es una reducción en el tiempo del procedimiento, dado que permite el entrenamiento quirúrgico, reducir el riesgo de infecciones y otros eventos que puedan ocurrir. Además, esta tecnología puede ser un recurso educativo (De Bona, 2019).

En áreas de la salud, la impresión 3D, prototipado rápido, se ha utilizado en medicina humana, odontología; medicina veterinaria, con la producción de biomodelos (De Alcantara, 2017). El objetivo es construir métodos alternativos para el estudio, en el campo veterinario, de la vía digestiva de los perros, logrando el mismo 
rendimiento y calidad en la enseñanza sin la necesidad de sacrificio de animales. Este sistema permite, al usuario, sentirse inmerso en el ambiente, estando cerca de una situación real. Además, el usuario podrá estudiar la anatomía y el funcionamiento del Sistema desde el interior, viéndolo con detalles (Ferreira et al., 2007).

En las últimas décadas, se ha desarrollado los escáneres láser, lo cual ha hecho posible capturar, de forma rápida, un conjunto de muestras suficientemente grande de los objetos de estudio. Con un escáner es posible obtener una distribución de puntos representantes de la superficie del objeto; en algunos casos información de color en los puntos. Para algunas aplicaciones es suficiente este conjunto de puntos como representación del objeto, haciendo la visualización directa de los puntos (Nobile, 2016). Por otra parte, la digitalización de cualquier objeto, por simple que sea, conlleva la realización de varias tomas con el escáner, las cuales dan lugar a varias nubes de puntos, que se deben fusionar en una única malla. Usualmente, el número de puntos suele ser excesivamente alto, debido a que el muestreo es fijo, e independiente de las irregularidades del objeto, cada uno de estos pasos se resuelve con procesos semiautomáticos, con un consumo alto de tiempo y recursos de cálculo (Nobile, 2016).

La enseñanza simultánea de la anatomía y la radiología ha demostrado resultados positivos, la representación de imágenes virtuales, por tomografía computarizada tridimensional (TC 3D), utilizada en un plan de estudios de cirugía, así como en la planificación quirúrgica para medicina, han potencializado el aprendizaje. Una ventaja es que la digitalización de elementos óseos permite conservar los elementos originales, evitando que estos deban ser reemplazados al cabo de un tiempo determinado; además, permite su acceso por parte de estudiantes, docentes, profesionales e investigadores de todo el mundo (Nobile, 2016).

Uno de los ejemplos actuales, por resaltar, es el de los modelos anatómicos del estómago de los perros. Se producen en masa y presentan características visualmente fieles a los órganos escaneados por una resonancia magnética 3D. Funcionan como réplicas realistas de esos órganos (Hackman et al., 2019) en estos modelos. Es posible identificar las diferentes regiones del estómago canino (aglandular y glandular); la anatomía regional del órgano (cardial, cuerpo y el antro pilórico); así como importantes características anatómicas de la especie, como las curvaturas mayores y menores del estómago. Además, se puede observar las relaciones topográficas del estómago con otros órganos como: el esófago, el duodeno descendente y el páncreas. Debido al hecho de que los modelos impresos se producen a partir de un material termoplástico, el uso, como material didáctico, no se limitaría a los laboratorios de anatomía; sino que, también, podría utilizarse en otros lugares como bibliotecas y aulas. Permitirá estudiar la anatomía del estómago en otros lugares, incluso en casa, no sólo en los laboratorios de anatomía. Los biomodelos son ligeros, resistentes y producidos con un material similar al plástico. Otro subproducto es la creación de archivos digitalizados, con la posibilidad de imprimir copias en cualquier momento (Hackman et al., 2019).

\section{Los modelos didácticos en cirugía}

Al analizar los fundamentos de la cirugía científica, se encuentra: la observación, la investigación clínica y la investigación experimental. Se puede realizarse en seres humanos, respetando los principios de la ética. sin embargo, en medicina veterinaria, la mayor parte de la investigación quirúrgica se lleva a cabo en animales de laboratorio (De la Garza-Rodea et al., 2007). Lo anterior ha llevado a muchos autores a referenciar que el uso de animales, para experimentar, es obligatorio. Con esto, los cirujanos adquieren el desarrollo de su competencia quirúrgica, lo cual hace que la cirugía experimental, en pro del diseño de modelos experimentales, vaya cobrando más fuerza en la actualidad (De La Garza-Rodea et al., 2007). 
La educación está sufriendo un proceso de cambio. El área de la medicina y la cirugía no se quedan atrás. Por ello, paradigmas antiguos, usados para la enseñanza tradicional, deben ser reemplazados por paradigmas modernos de tipo constructivista. Reyes et al. (2012) nos dicen que es una realidad que la educación está en una etapa transcendental de cambio; que requiere una participación más activa del alumno para que sea capaz de generar su propio conocimiento. Se debe recordar que el uso de simuladores en la enseñanza viene desde los años 20, cuando Edgar Link desarrolló los simuladores de vuelo para el entrenamiento de los pilotos (Molina \& Silveira, 2012). Sin embargo, hasta hace dos décadas, se empezó a utilizar, en medicina, y más específicamente en el área de anestesia (Molina\& Silveira, 2012), dando paso a que en la enseñanza de hoy cobre un papel importante para la integración entre las ciencias básicas y clínicas (Molina\& Silveira, 2012).

Si en verdad no se conocen muchos modelos inanimados para la cirugía tradicional, sí se cuenta con los modelos experimentales de otras especies animales para realizar prácticas, uno de ellos es el conejo. Halabi et al. (2012), afirman que, en la cirugía experimental se someten a prueba distintos procedimientos, tanto innovadores como tradicionales, estos últimos principalmente para docencia. Otros se realizan en animales de compañía, como el perro. Sin embargo, tienen restricciones de tipo ético y emocional (experimentar en una especie con la que existen lazos afectivos) que complicarían su uso como modelo en investigación. Otros autores, como Reyes et al. (2012), proponen el uso de la pata del cerdo como modelo biológico, pues resulta un recurso innovador; su fácil adquisición y preparación hace de él un modelo ideal.

Numerosos estudios, realizados a lo largo de los años, han logrado perfeccionar las técnicas quirúrgicas y la enseñanza a los alumnos. Mejora la curva de aprendizaje y en sí, el entrenamiento en las diferentes técnicas quirúrgicas. Desde este punto de vista los modelos experimentales inanimados empiezan a cobrar mucha importancia, hoy, existen muchos biomodelos inanimados de entrenamiento en cirugía de mínima invasión. Seguidamente, se destacan algunos estudios junto con el punto de vista de diferentes autores:

- Urson et al. (2006) afirman que antes de usar modelos experimentales animales, es bueno utilizar modelos inanimados para familiarizarse con el instrumental y la anatomía del animal.

- Mantrana et al. (2018) afirman que: los modelos hápticos, creados con impresoras 3D, han demostrado ser de gran utilidad en la planificación y ejecución de cirugías reparadoras complejas, al aportar grandes ventajas con su utilización.

- Dávila et al. (2008) afirman que: aunque todos los procedimientos quirúrgicos abdominales se pueden hacer, actualmente, por vía laparoscópica y, aunque existen nuevas y prometedoras tecnologías, como el sistema robótico, esto no significa el fin de la cirugía abierta, la cual seguirá desempeñando un papel importante.

- Dávila et al. (2008) refiere que las maniobras quirúrgicas básicas, que un estudiante de pregrado debería aprender durante la carrera de medicina, implican la adquisición de destrezas y habilidades manuales cruentas, tales como: suturas, drenajes (abscesos, seromas, hematomas) extracción de cuerpos extraños, etc. Estos procedimientos, por su carácter invasivo, significan una dificultad en el proceso tradicional de enseñanza-aprendizaje realizado al lado y sobre el paciente, dado que son difíciles de adquirir en la práctica clínica: siempre debe anteponerse la seguridad del paciente a las necesidades de la enseñanza. Como consecuencia de ello, sólo un pequeño grupo de alumnos, especialmente motivados y con acceso a ciertos servicios, puede llegar a obtener alguna experiencia en estas maniobras al momento de recibirse de médicos. Por consiguiente, en su estudio, diseñan un modelo experimental para simular y adquirir conocimientos y destrezas básicas en lo referente a la pared abdominal. 
La Universidad Complutense de Madrid (UCM) publicó, en internet, un proyecto de la Facultad de Veterinaria; específicamente del área de anatomía y embriología; desarrollado por (Gaspar et al., 2018), en el cual, este y otros autores, plantean objetivos que describen la utilización de modelos impresos en 3D para superar las barreras y las dificultades de hoy para la adquisición de cadáveres o la toxicidad de los conservantes. De esta forma, permite, a los alumnos, comprender la importancia de familiarizarse con estos modelos para favorecer el aprendizaje en el marco de la anatomía y la cirugía.

Actualmente, los autores deeste texto, en la Facultad de Medicina Veterinaria de UNIAGRARIA, están trabajando en un proyecto para la construcción de un biomodelo didáctico del sistema digestivo que sirva como herramienta en la simulación de diferentes procedimientos quirúrgicos (Figuras 2, 3, 4). El objetivo principal es implementar estas estructuras en las clases de pregrado y obtener la retroalimentación experiencial de los estudiantes, reforzando, así, la enseñanza tradicional con prácticas en cadáveres y pacientes de la clínica de pequeños animales.

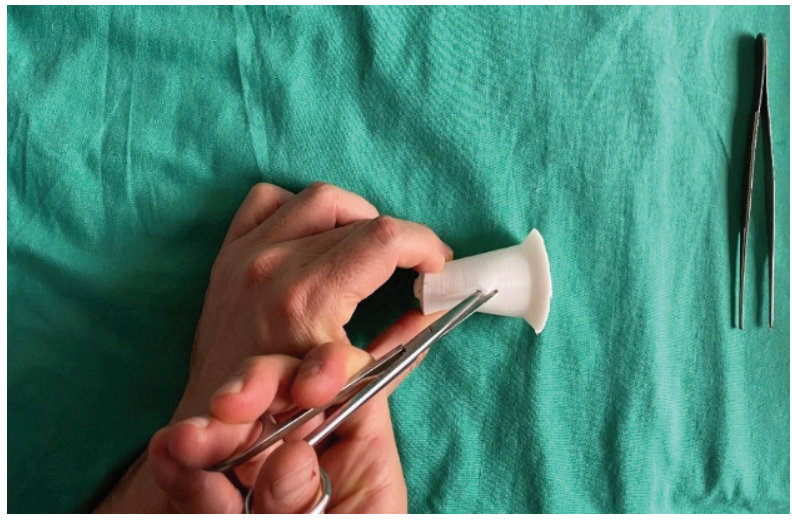

Figura 2. Manipulación de material Fuente autores

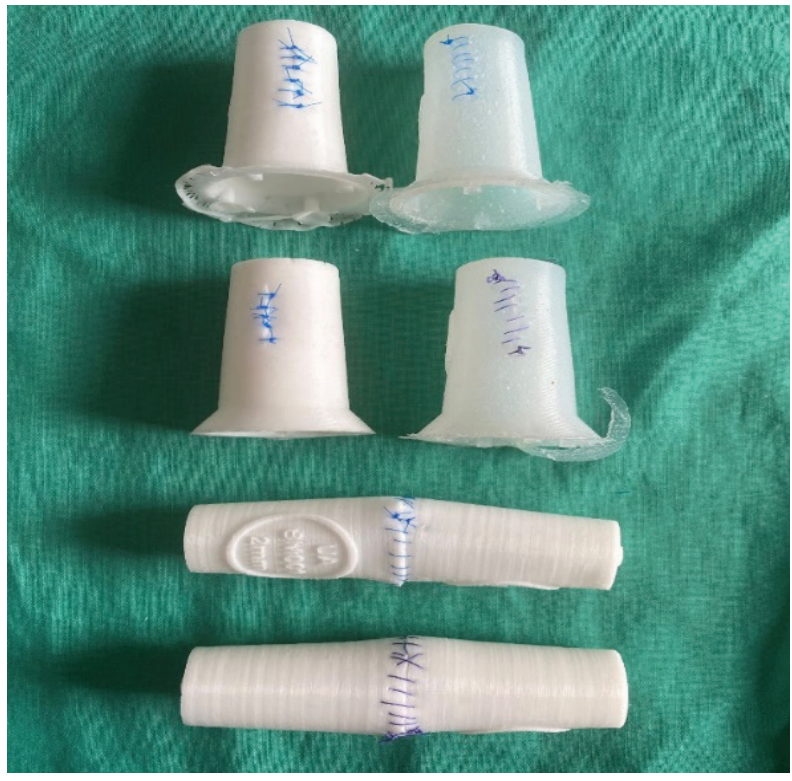

Figura 3. Aplicación Patrones de sutura. Fuente autores. 


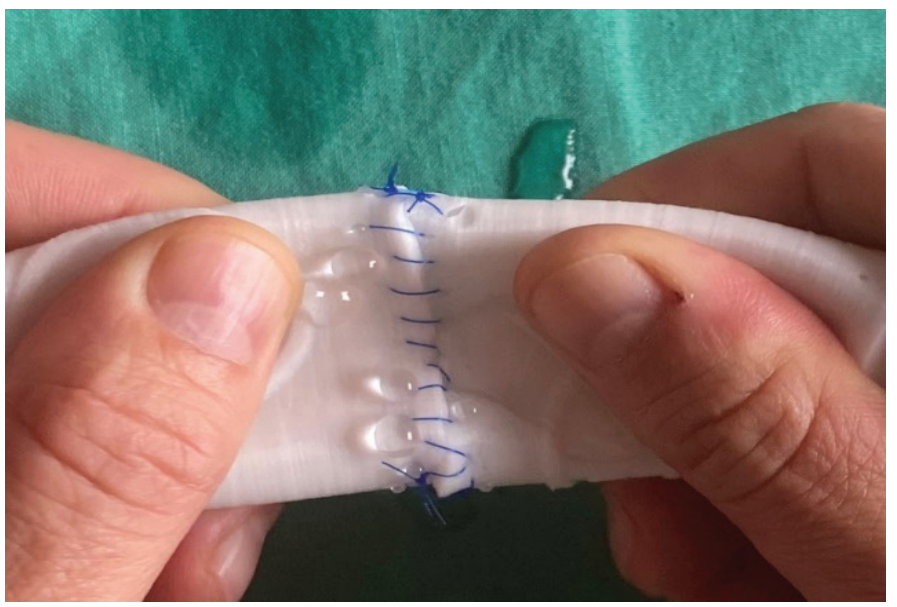

Figura 4. Realización de pruebas de fuga. Fuente Autores.

\section{Los modelos didácticos en patología}

La enseñanza de la patología se basa en dos grandes mecanismos de apreciación visual. Ambas encaminadas al diagnóstico. La evaluación de necropsia abarca un aspecto de descripción macroscópica, en la cual, el estudiante y el patólogo deben hacer un reconocimiento por patrones de lesión que conllevan a una aproximación diagnóstica, planteando así diagnósticos diferenciales. La segunda parte de la apreciación patológica es la histopatología, la cual, mediante el procesamiento de tejidos fijados en formol y el uso de microscopio, se logra llegar al detalle celular para explicar, a profundidad, las lesiones encontradas y ofrecer un diagnóstico aproximado o definitivo.

Se ha realizado estudios asociados con diversas patologías mediante modelos virtuales que pueden incluir muchas otras características de los tejidos lesionados: cambios de temperatura, color, lesión tisular, pueden ser ejemplo de ello al comparar los grados de aprendizaje (Lee et al., 2019). Los modelos tridimensionales, para enseñanza, tienen la característica de conservarse mejor en el tiempo; alta fidelidad en el modelado con la región anatómica, puesto que se pueden emplear tomografías y otras técnicas de detalle para usar el diseño de estos modelos. Es más fácil de almacenar si se compara con los tejidos fijados en formol; normalmente se usan en la enseñanza. Cada pieza modelada, tridimensionalmente, puede ser reemplazadas constantemente sobre un modelo previo; así no se estaría sujeto a enseñar aquellos casos que sean remitidos a un servicio diagnóstico y sujetos a su disponibilidad. Otra ventaja, importante, de la aplicación adecuada de los modelados en 3D, para la enseñanza de la patología, es el factor de seguridad, dado que, en gran medida, no hay que trabajar con cadáveres en enseñanza, al minimizar la exposición a patógenos, tanto por estudiantes como docentes; además de los posibles accidentes que podrían llegar a darse en el desarrollo de la técnica de necropsia (Amr \& Bennett, 2014; Inzunza et al., 2015; Jiménez et al., 2019).

Cuando se analizó la. construcción de un biomodelo didáctico (por ejemplo el del proyecto mencionado en UNIAGRARIA), un aspecto que se debe tener muy presente es que, si no se cuenta con el suficiente detalle anatómico para la enseñanza, estas falencias deberían ser suplementadas con herramientas anexas que permitan un mejor entendimiento y ampliación del tema o patología en revisión ( $\mathrm{Du}, 2016)$, en conjunto 
con la información adicional que involucra un paciente en su evolución clínica, juntos se pueden convertir en un instrumento de vital importancia en el desarrollo de habilidades clínicas interpretativas en el estudiante. Adicionalmente, aspectos como el peristaltismo, capacidad de dilatación y deformidad del material usado para representar el intestino es un factor fundamental para tener presente, para este último punto y con el fin de generar una experiencia de enseñanza aún más interactiva, algunos autores han planteado el uso de nuevas tecnologías como implementación de gafas de realidad virtual o realidad aumentada, que podría llegar a ser una posible aplicación para considerar a futuro, puesto que el grado de representación de diversas situaciones y abordajes clínico-patológicos son muy exactos y válidos como experiencia práctica (Hanna et al., 2018).

\section{Conclusión}

El uso de los biomodelos didácticos es de suma importancia para la formación de médicos veterinarios y profesionales de la salud. Debido a cuestiones: éticas, morales, ambientales y financieras; el estudio de la anatomía, fisiología, patología o cirugía, a través de cadáveres y animales vivos, está en discusión. Por tanto, es fundamental desarrollar métodos alternativos para el estudio de estas disciplinas, permitiendo su continuidad, dado su debido papel en la formación de los estudiantes. Mediante el uso de modelos tridimensionales, de distintas áreas de la medicina en diversas especies, se puede implementar la enseñanza práctica simulada, con el fin de otorgar nociones mínimas aplicadas a un problema o abordaje en específico, al adquirir destreza y un análisis adecuado al momento de enfrentar, en la vida profesional, esas situaciones previamente simuladas. Los resultados de este tipo de proyectos demuestran, claramente, que la digitalización y la impresión 3D contribuyen, fuertemente, al aprendizaje. Actualmente juegan un papel importante mediante la producción de réplicas que presentan características deseables de los órganos y los tejidos. En gran medida, el uso reiterativo de ese tipo de ayudas podría aplicarse en procedimientos que deben ser mecanizados; con ciertos pasos para su correcta aplicación, en lo posible conociendo y simulando condiciones clínicas, técnicas quirúrgicas entre otras, que buscan dar herramientas al médico para que, en su ejercicio profesional, tenga una mayor capacidad y autonomía para la toma de decisiones al momento de enfrentarse condiciones de urgencia o cotidianidad. Por consiguiente, el uso de modelos didácticos de estudio son una útil alternativa al uso de animales vivos en áreas donde pueda llegar a ser aplicado, reforzando las clases teóricas y prácticas en pregrado y asimismo, promoviendo el proceso de enseñanza aprendizaje.

\section{Conflicto de intereses}

Los autores declaran no tener ningún conflicto de intereses potencial con respecto a la investigación, la autoría, y / o publicación de este artículo.

\section{Referencias}

Amr, M., \& Bennett, M. (2014). Introducing 3-Dimensional Printing of a Human Anatomic Pathology Specimen Potential Benefits for Undergraduate and Postgraduate Education and Anatomic Pathology Practice. Archives of Pathology $\sigma^{2}$ Laboratory Medicine. 139(8):1048-51. doi: 10.5858/arpa.2014-0408-OA

Anandit, J., Niranjini, C., \& Vinay, O. (2018). All play and no work: skits and models in teaching skeletal muscle Physiology. Advances in Physiology Education 42: 242-246. doi: 10.1152/advan.00163.2017 
Dávila, F., Moreno, A., Rivera, J., \& Rojas, P. (2008). Simulador de pared abdominal para adquisición de habilidades básicas de cirugía. Revista Mexicana de Cirugía Endoscópica. 9(2): 66-70. https://www. medigraphic.com/pdfs/endosco/ce-2008/ce082e.pdf

De Alcântara, B. (2017). Biomodelos Ósseos Produzidos por Intermédio da Impressão 3D: Uma Alternativa Metodológica no Ensino da Anatomia Veterinária. Revista De Graduação USP. 47-53. doi: 10.11606/ issn.2525-376X.v2i3p47-53

De Bona, C. (2019). Trabalho de Conclusão de Curso aprendizagem de anatomia vertebral humana por meio do uso de modelos vertebrais lombares $2 \mathrm{~d}$ e $3 \mathrm{~d}$. Instituto federal de educação, ciência e tecnologica de santa catarina - câmpus florianópolis, florianópolis. https://repositorio.ifsc.edu.br/bitstream/ handle/123456789/1040/TCC\%20\%20-\%20vers\%C3\%A3o\%20final\%20Cristiane\%20De\%20Bonaconvertido.pdf?sequence $=1$

De la Garza-Rodea, A., Padilla-Sánchez, L., De la Garza-Aguilar, J., \& Neri-Vela, R. (2007). Algunas notas sobre la historia del laboratorio de cirugía experimental. Reflexiones sobre su importancia en la educación e investigación quirúrgica. Cirugía y Cirujanos. 75(6): 499-505. https://www.medigraphic. com/pdfs/circir/cc-2007/cc0760.pdf

DiCarlo, S. (2013). Student construction of anatomic models for learning complex, seldom seen structures. Advances in Physiology Education 37: 440-441. doi: doi.org/10.1152/advan.00098.2013

Du, P. (2016). The virtual intestine: in silico modeling of small intestinal electrophysiology and motility and the applications. WIREs Syst. Biol. Med. 8: 69-85. doi: 10.1002/wsbm.1324

Ferreira, E., Toledo, M., \& Hermosilla, L. (2007). Ferramenta didática para o ensino do aparelho digestivo canino utilizando técnicas de realidade virtual. Revista cientifica eletônica de psicologia . 7: 1-7.

Forero, A. (2016). Diseño de material didáctico para la enseñanza de anatomía, IFDP`16 - Systems E̋ Design: Beyond Processes and Thinking. Editorial Universitat Politècnica de València. 1015-1030. Doi: 10.4995/ IFDP.2015.2955

Friederichs, H., Weissenstein, A., Ligges, S., Möller, D., Becker, J.C., \& Marschall, B. (2014). Combining simulated patients and simulators: pilot study of hybrid simulation in teaching cardiac auscultation. Advances in Physiology Education 38: 343-347. doi: 10.1152/advan.00039.2013

Gaspar, S., Rojo, I., \& Salvador, C. 2018. Virtualización e impresión 3D de modelos anatómicos aplicados a la docencia en anatomía y cirugía veterinaria II. Universidad Complutense de Madrid. 123: 1 - 11. https://eprints.ucm.es/48081/

Gómez, A., Ramírez, A., \& Cortés, J. (2015). Biomodelos animales y enfermedades infecciosas de importancia en salud pública veterinaria. Revista Sapuvet de Salud Pública. 2: 69-94. https://www.researchgate. net/publication/274312271_Biomodelos_animales_y_enfermedades_infecciosas_de importancia en_salud_publica_veterinaria

González, M., Lara, P., \& González, J. (2015). Modelos educativos en medicina Modelos educativos en medicina y su evolución histórica. Rev. Esp. Méd. Quir. 20: 256-265. https://www.medigraphic.com/ pdfs/quirurgicas/rmq-2015/rmq152v.pdf 
Gookin, J., McWhorter, D., Vaden, S., \& Posner, L. (2010). Outcome assessment of a computer-animated model for learning about the regulation of glomerular filtration rate. Advances in Physiology Education 34: 97-105. doi: 10.1152/advan.00012.2010

Hackmann, C., Dos Reis, D., \& Chaves De-Assis-Neto, A. (2019). Digital Revolution In Veterinary Anatomy: Confection of Anatomical Models of Canine Stomach by Scanning and Three-Dimensional Printing (3D). International Journal of Morphology, 37(2): 1 - 4. http://www.intjmorphol.com/wp-content/ uploads/2019/04/art_16 372.pdf

Halabi, T., Bahamondes, F., \& Cattaneo, G. (2012). Estómago de Conejo: Modelo Animal para Cirugía experimental. International Journal of Morphology. 30(1): 82-87. doi: 10.4067/S0717$\underline{95022012000100014}$

Hanna, M., Ahmed, I., Nine, J., Prajapati, S., \& Pantanowitz, L. (2018). Augmented Reality Technology Using Microsoft HoloLens in Anatomic Pathology. Archives of Pathology $\sigma^{2}$ Laboratory Medicine. 142(5): 638-644. Doi: 10.5858/arpa.2017-0189-OA

Inzunza, O., Caro, I., Mondragón, G., \& Baeza, F. (2015). Impresiones 3D, nueva tecnología que apoya la docencia anatómica. International Journal of Morphology 33(3): 1176-1182. Doi: 10.4067/S0717$\underline{95022015000300059}$

Izaguirre, T., Jeremías, R., \& Izaguirre, J. (2001). Técnicas avanzadas en recuperación de tejidos orgánicos y su aplicación en la docencia actual. Gaceta Médica Caracas. 109(1): 36-39.

Jiménez, G., Tobón, H., \& Vélez, A. (2019). Uso de la simulación en la enseñanza de la patología quirúrgica. Revista Salud Bosque. 9 (2): 56-64. Doi: 10.18270/rsb.v9i2.2808

Kuebler, W., Mertens, M., \& Pries, A. (2007). A two-component simulation model to teach respiratory mechanics. Advances in Physiology Education 31: 218-222. Doi: 10.1152/advan.00001.2007

Lee, J. (2010). Three-dimensional computed tomographic volume rendering imaging as a teaching tool in veterinary radiology instruction. Veterinarni Medicina. 603-609 . http://vri.cz/docs/ vetmed/55-12-603.pdf

Lee, B.C., Hsieh, S.T., Chang, Y.L., Tseng, F.Y., Lin, Y.J., Chen, Y.L., Wang, S.H., Chang, Y.F., Ho, Y.L., Ni, Y.H., \& Chang, S.C. (2020). A Web-Based Virtual Microscopy Platform for Improving Academic Performance in Histology and Pathology Laboratory Courses: A Pilot Study. Anatomical Sciences Education 13 (6):1-16. doi: $\underline{10.1002 / a s e .1940}$

Lenis, Y., \& Arango, T. (2011). Modelos didáticos como iniciativa para o ensino da anatomia e fisiologia animal. Journal of Morphological Sciences. 20: $44-51$.

Lujan, H., Krishnan, S., O’Sullivan, D., Hermiz, D., \& Janbaih, H. (2000). La experimentación animal y el desarrollo de la investigación. Revista Hispanoamericana Anim Exp. 5(4): 9 -17.

Mantrana, G., JAcobo, O., \& Hartwing, D. (2018). Three-Dimensional printing models in the preoperative planning and academic education of mandible fractures. Cirugia Plástica Ibero-Latinoamericana. 44(2): 193-201. doi: $10.4321 /$ S0376-78922018000200010 
Massari, C., Pinto, A., De Carvalho, Y., \& Silva, A. (2019). Volumetric Computed Tomography Reconstruction, Rapid Prototyping and 3D Printing of Opossum Head (Didelphis albiventris). International Journal of Morphology 37(3): 838-844. http://www.intjmorphol.com/wp-content/ uploads/2019/07/art_09 373.pdf

Molento, C., \& Calderón, N. (2009). Essential directions for teaching animal welfare in South America. Rev. sci. tech. Off. Int. Epiz. 28(2): 617-625. https://www.researchgate.net/profile/Carla_Molento/ publication/41403452_Essential_directions_for_teaching_animal_welfare_in_South_America/ links/0912f50908203d3061000000.pdf

Molina, J., 6 Silveira, P. (2012). Los simuladores y los modelos experimentales en el desarrollo de habilidades quirúrgicas en el proceso de enseñanza-aprendizaje de las Ciencias de la Salud. REDVET, 13(6): 1-23. https://www.redalyc.org/pdf/636/63624434013.pdf

Nobile, N. (2016). Biblioteca Digital 3D de Huesos Humanos. Universidad Nacional de Cordoba. 1: 1-58.

Rodenbaugh, D., Lujan, H., \& DiCarlo, S. (2012). Learning by doing: construction and manipulation of a skeletal muscle model during lecture. Advances in Physiology Education 36: 302-306. doi: 10.1152/ advan.00093.2012

Sajal, C., Bhaskar, A., \& Oommen, V. (2018). Pumping the pulse: a bicycle pump to simulate the arterial pulse waveform. Advances in Physiology Education 42: 256-259. doi: 10.1152/advan.00004.2018

Satheesha, N., \& Soumya, A. (2009). A simple model to demonstrate the movements and the axes of the eyeball. Advances in Physiology Education 33: 356-357. Doi: 10.1152/advan.00047.2009

Usón, G., Sánchez, M., \& Díaz-Güemes, M. (2006). Modelos experimentales en la cirugía laparoscópica urológica. Actas Urológicas Españolas. 30(5): 443-450. doi: 10.1016/S0210-4806(06)73478-7

Villamizar, J., \& Aquino, A. (2016). Experimentación con biomodelos animales en ciencias de la salud. Avances en Biomedicina, Revista Saber Ula. 5 (3): 173-177. http://erevistas.saber.ula.ve/index.php/ biomedicina/article/view/7980/7927

Reyes-Arellano, W., Tapia-Jurado, J., Cortes-González, J., Jiménez-Corona, J., Delgado-Reyes, L., \& Montalvo-Javé, E. (2012). Modelo biológico de enseñanza para la extirpación de lipoma. Revista Médica del Hospital General de México. 75(4) 247 - 253. https://www.elsevier.es/en-revista-revista-medica-delhospital-general-325-articulo-modelo-biologico-ensenanza-extirpacion-lipoma-X0185106312842605 